\title{
Estudo Comparativo de Antieméticos e Suas Associações, na Prevenção de Náuseas e Vômitos Pós-Operatórios, em Pacientes Submetidas a Procedimentos Cirúrgicos Ginecológicos*
}

\author{
Comparative Study of Anti-Emetics and Their Association, in the \\ Prevention of Postoperative Nausea and Vomiting in Patients \\ Undergoing Gynecologic Surgeries
}

Taylor Brandão Schnaider', Antônio Mauro Vieira, TSA², Antônio Carlos Aguiar Brandão, TSA ${ }^{3}$

\section{RESUMO}

Schnaider TB, Vieira AM, Brandão ACA - Estudo Comparativo de Antieméticos e Suas Associações, na Prevenção de Náuseas e Vômitos Pós-Operatórios, em Pacientes Submetidas a Procedimentos Cirúrgicos Ginecológicos.

JUSTIFICATIVA E OBJETIVOS: A profilaxia de náuseas e vômitos pósoperatórios foi objeto de muitos estudos. O objetivo desta pesquisa foi comparar antieméticos e associações na prevenção de náuseas e vômitos pós-operatórios.

MÉTODO: Setenta pacientes, ASA I e II, foram submetidas a procedimentos cirúrgicos ginecológicos, sob bloqueio peridural associado à anestesia geral. No Grupo Metoclopramida (GM), administrou-se $20 \mathrm{mg}$; no Grupo Dexametasona (GDe), injetou-se $8 \mathrm{mg}$; no Grupo Droperidol (GDr) administrou-se 1,25 mg; no Grupo Ondansetron (GO) injetou-se $8 \mathrm{mg}$; no Grupo Dexametasona-Ondansetron (GDeO) administrou-se, respectivamente, $8 \mathrm{mg}$ e $4 \mathrm{mg}$; no Grupo Droperidol-Ondansetron (GDrO) injetou-se 1,25 mg e $4 \mathrm{mg}$; no Grupo Dexametasona-DroperidolOndansetron (GDeDrO) administrou-se $8 \mathrm{mg}, 0,625 \mathrm{mg}$ e $4 \mathrm{mg}$. A presença de náuseas e vômitos foi observada nos momentos de 6, 12, 24 e 36 horas após o término do ato operatório.

RESULTADOS: A incidência total de episódios de náuseas foi de quatro no GDeDrO, seis no GO, seis no GDrO, 11 no GDe, 11 no GDeO, 18 no GM e 22 no GDr. Aplicando-se o teste do Qui-quadrado ou o teste de Fisher, ocorreu diferença estatística significativa entre o $\mathrm{GDr}$ e os grupos GDe, GDO, GDrO, GDeO, GDeDrO; entre o GM e os grupos GO, GDrO e GDeDrO; entre o GDeO e o grupo GDeDrO. A inci-

${ }^{*}$ Recebido do (Received from) Centro de Ensino e Treinamento do Serviço de Anestesiologia do Hospital das Clínicas da Faculdade de Ciências Médicas de Pouso Alegre (HC-FCM-UNIVAS), Pouso Alegre, MG

1. Professor Titular Doutor da UNIVAS; Responsável pelas Disciplinas de Anestesiologia e Metodologia Científica; Co-Coordenador do DINTER UNIFESP/UNIVAS

2. Professor Titular Doutor da UNIVAS; Responsável pela Disciplina de Farmacologia; Co-Responsável pelo CET/SBA de Pouso Alegre

3. Professor Titular Doutor da UNIVAS; Responsável pela Disciplina de Biofísica; Responsável pelo CET/SBA de Pouso Alegre

Apresentado (Submitted) em 12 de fevereiro de 2008 Aceito (Accepted) para publicação em 18 de agosto de 2008

Endereço para correspondência (Correspondence to):

Dr. Taylor Brandão Schnaider

Av. Francisca Ricardina de Paula, 289 - Medicina

37550-000 Pouso Alegre, MG

E-mail: sormanti@uai.com.br

() Sociedade Brasileira de Anestesiologia, 2008 dência total de episódios de vômitos foi de três no GO, três no GDeDrO, seis no GDrO, sete no GDe, sete no GDeO, desz no GDr e 13 no G. Verificou-se diferença estatística significativa entre o GDr e os grupos GO e GDeDrO; entre o GM e os grupos GO e GDeDrO.

CONCLUSÕES: A associação dexametasona-droperidol-ondansetron e o ondansetron foram mais eficazes na profilaxia de náuseas e vômitos.

Unitermos: ANTIEMÉTICOS: dexametasona, droperidol, metoclopramida, ondansetron; CIRURGIA: Ginecológica; COMPLICAÇÕES: náuseas, vômitos.

\section{SUMMARY}

Schnaider TB, Vieira AM, Brandão ACA - Comparative Study of Antiemetics and their Association, in the Prevention of Postoperative Nausea and Vomiting in Patients Undergoing Gynecologic Surgeries.

BACKGROUND AND OBJECTIVES: Prophylaxis of postoperative nausea and vomiting has been the subject of several studies. The objective of the present study was to compare anti-emetics, and their association, in the prevention of postoperative nausea and vomiting.

METHODS: Seventy patients, ASA I and II, underwent epidural block associated with general anesthesia for gynecologic surgeries. Patients in the Metochlopramide Group (MG) received $20 \mathrm{mg}$ of the drug; the Dexamethasone Group (DeG) received $8 \mathrm{mg}$; the Droperidol Group (DrG) received $1.25 \mathrm{mg}$; the Ondansetron Group (OG) received $8 \mathrm{mg}$; the Dexamethasone-Ondansetron Group (DeOG) received $8 \mathrm{mg}$ and $4 \mathrm{mg}$, respectively; the Droperidol-Ondansetron Group (DrOG) received 1.25 $\mathrm{mg}$ and $4 \mathrm{mg}$, respectively; the Dexamethasone-Droperidol-Ondansetron Group (DeDrOG) received $8 \mathrm{mg}, 0.625 \mathrm{mg}$, and $4 \mathrm{mg}$. The presence of nausea and vomiting was evaluated at $6,12,24$, and 36 hours after the end of the surgery.

RESULTS: The total incidence of episodes of nausea per group is as follows: 4 in DeDrOG, 6 in OG, 6 in DrOG, 11 in DeG, 11 in DeOG, 18 in MG, and 22 in DrG. The Chi-square and Fisher exact tests indicated statistically significant differences between DrG and DeG, DOG, DrOG, DeOG, and DeDrOG; between MG and OG, DrOG, and DeDrOG; and between DeOG and DeDrOG. And the incidence of vomiting was: 3 in OG, 3 in DeDrOG, 6 in DrOG, 7 in DeG, 7 in DeOG, and 10 in DrG, and 13 in $G$. There was a statistically significant difference between DrG and OG and DeDrOG; and between MG and OG and DeDrOG.

CONCLUSIONS: The association dexamethasone-droperidol-ondansetron and ondansetron alone were more effective in the prophylaxis of nausea and vomiting.

Key Words: ANTI-EMETICS: dexamethasone, droperidol, metochlopramide, ondansetron; COMPLICATIONS: nausea, vomiting; SURGERY: gynecologic. 


\section{INTRODUÇÃO}

Certos odores, a desidratação, a dor, a apreensão e o medo contribuem para náuseas e vômitos pós-operatórios ${ }^{1}$. Além disso, náuseas e vômitos contribuem para aumentar os custos nosocomiais, retardando as altas hospitalares, exigindo internações não-esperadas, acarretando maior atenção da equipe médica e um menor grau de satisfação do paciente ${ }^{2}$. Outras complicações decorrentes são aspiração de vômito, pneumonia aspirativa, distúrbios hidroeletrolíticos, rutura esofágica, deiscência de sutura cirúrgica e aumento da pressão intracraniana ${ }^{2}$.

A etiologia dos episódios de náuseas e vômitos pode estar relacionada com o paciente (sexo, idade, peso, ansiedade, história prévia de náuseas e vômitos pós-operatórios e tabagismo), com a técnica anestésica (óxido nitroso, halogenados e opióides) e com o local e a duração do ato operatório ${ }^{3,4}$.

O gênero feminino e o procedimento cirúrgico ginecológico são fatores de risco importantes, com incidência em torno de $70 \%$ de náuseas e vômitos no pós-operatório. Por causa dessa incidência, têm-se buscado encontrar o antiemético ideal para essas pacientes ${ }^{2}$.

A metoclopramida e o droperidol, ambos antagonistas dos receptores dopaminérgicos, têm sido efetivos na prevenção e no tratamento de náuseas e vômitos pós-operatórios, mas podem causar sedação, disforia, síndrome extrapiramidal e torcicolo espasmódico ${ }^{2,5}$.

A dexametasona, um corticosteróide com efeito antiemético desconhecido, também tem sido efetiva na prevenção de náuseas e vômitos pós-operatórios, porém com mais eficiência se associada a outros antieméticos ${ }^{2,5}$.

A ondansetrona, um antagonista dos receptores serotoninérgicos (receptor 5-HT3), tem sido a mais aceita para a prevenção de náuseas e vômitos pós-operatórios em pacientes com mais fatores de risco ${ }^{2,5}$.

Antieméticos com diferentes mecanismos de ação apresentam ações sinérgicas quando combinados entre si, resultando em mais eficácia na prevenção e no tratamento de náuseas e vômitos pós-operatórios ${ }^{2,5-9}$.

Em decorrência dos freqüentes episódios de náuseas e vômitos pós-operatórios e da evolução do arsenal farmacêutico anestésico e antiemético, o objetivo desta pesquisa foi comparar antieméticos, administrados isoladamente ou associados, na prevenção desses efeitos adversos, em procedimentos cirúrgicos ginecológicos.

\section{MÉTODO}

Após aprovação do projeto pelo Comitê de Ética em Pesquisa em Seres Humanos da Universidade do Vale do Sapucaí, todos os pacientes participantes assinaram o Termo de Consentimento Livre e Esclarecido, concordando com os procedimentos a que foram submetidos. Participaram des- te estudo clínico prospectivo e analítico, 70 pacientes do gênero feminino, na faixa etária de 20 a 50 anos, estado físico ASA I e II, submetidas a histerectomia total abdominal com anexectomia, sob anestesia geral associada à peridural. Todas as pacientes receberam diazepam $(10 \mathrm{mg})$ na véspera e midazolam (15 mg) 40 minutos antes do ato cirúrgico, ambos administrados por via oral. Na sala cirúrgica foram monitorizadas com eletrocardioscópio, pressão arterial nãoinvasiva, oxímetro de pulso e analisador de gases ins- e expirados. Depois de realizada a punção venosa com cânula 18G, foi administrada solução contendo midazolam (5 mg) e fentanil $(50 \mu \mathrm{g})$.

A seguir, foi infundido o antiemético ou suas associações, diluídos em $500 \mathrm{~mL}$ de cloreto de sódio a 0,9\%, antes da realização da punção peridural. No Grupo Metoclopramida (GM), foram administrados $20 \mathrm{mg}$; no Grupo Dexametasona (GDe), foram injetados $8 \mathrm{mg}$; no Grupo Droperidol (GDr), foram administrados 1,25 mg; no Grupo Ondansetron (GO), foram injetados $8 \mathrm{mg}$; no Grupo Dexametasona-Ondansetron (GDeO), foram administrados, respectivamente, $8 \mathrm{mg}$ e $4 \mathrm{mg}$; no Grupo Droperidol-Ondansetron (GDrO), foram injetados, respectivamente, 1,25 mg e $4 \mathrm{mg}$; no Grupo DexametasonaDroperidol-Ondansetron (GDeDrO), foram administrados, respectivamente, $8 \mathrm{mg}, 0,625 \mathrm{mg} \mathrm{e} 4 \mathrm{mg}$.

A anestesia peridural foi realizada com a paciente na posição sentada, punção no espaço $L_{2}-L_{3}$, com agulha Tuohy $15 \mathrm{G}$, sendo administrados $20 \mathrm{~mL}$ de ropivacaína a $0,75 \%$, na velocidade de $1 \mathrm{~mL} . \mathrm{s}^{-1}$.

A indução da anestesia geral foi realizada com etomidato $\left(0,2 \mathrm{mg} \cdot \mathrm{kg}^{-1}\right)$, alfentanil $\left(30 \mu \mathrm{g} \cdot \mathrm{kg}^{-1}\right)$ e rocurônio $\left(0,6 \mathrm{mg} \cdot \mathrm{kg}^{-1}\right)$, sendo a manutenção obtida pela administração de isoflurano ( 0,5 vol\% a 3,0 vol\%). Quando ocorreram sinais clínicos ou respostas hemodinâmicas que sugeriam níveis inadequados de anestesia (sudorese, lacrimejamento, hipertensão arterial e taquicardia) foi administrado alfentanil (500 $\mu \mathrm{g}$ ), em doses intermitentes, por via venosa.

A ventilação controlada foi efetuada em sistema de anestesia com baixo fluxo, possibilitando umidificação e aquecimento dos gases inspirados. $O$ volume corrente foi de 8 a $10 \mathrm{~mL}^{\mathrm{kg}} \mathrm{kg}^{-1} \mathrm{e}$ a freqüência respiratória suficiente para manter a pressão expirada de dióxido de carbono $\left(\mathrm{P}_{\mathrm{ET}} \mathrm{CO}_{2}\right)$ entre $30 \mathrm{mmHg}$ e $35 \mathrm{mmHg}$.

Os dados da pressão arterial, freqüência cardíaca, saturação periférica da hemoglobina pelo oxigênio $\left(\mathrm{SpO}_{2}\right)$, gás carbônico expirado $\left(\mathrm{P}_{\mathrm{ET}} \mathrm{CO}_{2}\right)$ e concentração inspirada de isoflurano foram registrados após a monitorização, a realização da anestesia peridural, a intubação traqueal e, a seguir, de 15 em 15 minutos até o término do ato cirúrgico. Não foi realizado antagonismo do bloqueio neuromuscular em nenhuma das pacientes no final do procedimento cirúrgico.

Encerrado o procedimento anestésico-cirúrgico, as pacientes foram encaminhadas à sala de recuperação pós-anestésica (SRPA). 
A incidência de náuseas e vômitos foi observada nos momentos de $6,12,24$ e 36 horas após o término do ato operatório.

Para analgesia pós-operatória foi administrada dipirona, um derivado pirazolônico, na dose de $15 \mathrm{mg} \cdot \mathrm{kg}^{-1}$, de quatro em quatro horas, após o término do procedimento cirúrgico.

A análise estatística relativa aos dados antropométricos das pacientes foi efetuada utilizando-se a Análise de Variância com a prova de Scheffé; a análise estatística do tempo de duração do ato operatório foi feita por meio do teste $t$ de Student; a análise da presença de náuseas e vômitos pós-operatórios foi efetuada utilizando-se os testes do Qui-quadrado e Exato de Fisher. Valores de $p<0,05$ foram considerados significativos.

\section{RESULTADOS}

No que se refere ao peso e à idade das pacientes incluídas no estudo, utilizando-se a Análise de Variância com a prova de Scheffé, não foram encontradas diferenças estatísticas significativas entre os grupos (Tabela I).
Quanto ao tempo de duração do ato cirúrgico, utilizando-se o teste $t$ de Student, não foram encontradas diferenças estatísticas significativas entre os grupos (Tabela II).

Não houve necessidade de dose suplementar de opióide para as participantes do experimento, tendo sido administrada somente a dose preconizada para a indução.

No que se refere à presença de náuseas, utilizando o teste do Qui-quadrado, observou-se diferença estatística significativa: entre o Grupo Droperidol e os grupos Dexametasona, Ondansetron, Droperidol-ondansetron, Dexametasona-ondansetron e Dexametasona-droperidol-ondansetron; entre o Grupo Metoclopramida e os grupos Ondansetron, Droperidol-ondansetron e Dexametasona-droperidol-ondansetron; entre o Grupo Dexametasona-ondansetron e o Grupo Dexametasona-droperidol-ondansetron (Tabela III).

Com relação à presença de vômitos, utilizando-se o teste Exato de Fisher, observou-se diferença estatística significativa: entre o Grupo Droperidol e os grupos Ondansetron e Dexametasona-droperidol-ondansetron; entre o Grupo Metoclopramida e os grupos Ondansetron e Dexametasonadroperidol-ondansetron (Tabela IV).

Tabela I - Dados Antropométricos das Pacientes Incluídas no Estudo

\begin{tabular}{lcc}
\hline & Idade (anos) & Peso $(\mathrm{kg})$ \\
\hline Grupo dexametasona $(n=10)$ & $44,5 \pm 4,97$ & $68,7 \pm 8,68$ \\
Grupo droperidol $(n=10)$ & $40,6 \pm 6,56$ & $68,4 \pm 7,86$ \\
Grupo ondansetron $(n=10)$ & $41,9 \pm 6,36$ & $68,3 \pm 10,04$ \\
Grupo metoclopramida $(n=10)$ & $41,8 \pm 6,35$ & $65,9 \pm 10,59$ \\
Grupo droperidol-ondansetron $(n=10)$ & $43,7 \pm 5,33$ & $58,5 \pm 10,26$ \\
Grupo dexametasona-ondansetron $(n=10)$ & $43,8 \pm 5,63$ & $63,8 \pm 12,04$ \\
Grupo dexametasona-droperidol-ondansetron $(n=10)$ & $41,4 \pm 6,36$ & $60,7 \pm 5,22$ \\
\hline
\end{tabular}

Valores expressos em Média \pm DP.

Sem significância estatística, prova de Scheffé, $p>0,05$.

Tabela II - Tempo de Duração do Ato Operatório

\begin{tabular}{lc}
\hline & Duração (min) \\
\hline Grupo dexametasona $(n=10)$ & $100 \pm 28$ \\
Grupo droperidol $(n=10)$ & $124 \pm 31$ \\
Grupo ondansetron $(n=10)$ & $110 \pm 25$ \\
Grupo metoclopramida $(n=10)$ & $108 \pm 26$ \\
Grupo droperidol-ondansetron $(n=10)$ & $88 \pm 40$ \\
Grupo dexametasona-ondansetron $(n=10)$ & $107 \pm 24$ \\
Grupo dexametasona-droperidol-ondansetron $(n=10)$ & $96 \pm 26$ \\
\hline
\end{tabular}

Valores expressos em Média \pm DP.

Sem significância estatística, teste $t$ de Student, $p>0,05$ 


\section{ESTUDO COMPARATIVO DE ANTIEMÉTICOS E SUAS ASSOCIAÇÕES, NA PREVENÇÃO DE NÁUSEAS E VÔMITOS PÓS-OPERATÓRIOS EM PACIENTES SUBMETIDAS A PROCEDIMENTOS CIRÚRGICOS GINECOLÓGICOS}

Tabela III — Pacientes com Náuseas nos Grupos de Estudo

\begin{tabular}{|c|c|c|c|c|c|c|c|}
\hline \multirow[b]{2}{*}{ Náusea } & \multicolumn{7}{|c|}{ Grupos } \\
\hline & $\mathrm{Dr}$ & $\mathrm{De}$ & M & $\mathrm{O}$ & DrO & $\mathrm{DeO}$ & DeDrO \\
\hline Até 6h & 9 & 5 & 7 & 4 & 4 & 4 & 1 \\
\hline $6 \mathrm{~h}$ a $12 \mathrm{~h}$ & 8 & 3 & 5 & 1 & 1 & 5 & 1 \\
\hline $12 \mathrm{~h}$ a $24 \mathrm{~h}$ & 3 & 3 & 5 & 1 & 1 & 2 & 1 \\
\hline $24 h$ a $36 h$ & 2 & 0 & 1 & 0 & 0 & 0 & 1 \\
\hline
\end{tabular}

Grupo: Dr - Droperidol; De - Dexametasona; M - Metoclopramida; O - Ondansetron; DrO - Droperidol-Ondansetron; DrO - DroperidolOndansetron; DeO - Dexametasona-Ondansetron; DeDrO - Dexametasona-Droperidol-Ondansetron.

* Diferença estatística significativa, teste do Qui-quadrado, $p<0,05$.

+ Diferença estatística significativa, teste do Qui-quadrado, $p<0,05$.

\# Diferença estatística significativa, teste do Qui-quadrado, $p<0,05$.

Tabela IV — Pacientes com Vômitos nos Grupos de Estudo

\begin{tabular}{|c|c|c|c|c|c|c|c|}
\hline \multirow[b]{2}{*}{ Vômitos } & \multicolumn{7}{|c|}{ Grupos } \\
\hline & $\mathrm{Dr}$ & $\mathrm{De}$ & M & 0 & DrO & $\mathrm{DeO}$ & DeDrO \\
\hline Até $6 \mathrm{~h}$ & 6 & 2 & 5 & 1 & 4 & 1 & 0 \\
\hline $6 h-12 h$ & 4 & 3 & 4 & 1 & 1 & 4 & 1 \\
\hline $12 h-24 h$ & 0 & 2 & 4 & 1 & 1 & 2 & 1 \\
\hline
\end{tabular}

Grupo: Dr — Droperidol; De — Dexametasona; M — Metoclopramida; O — Ondansetron; DrO — Droperidol — Ondansetron; DeO — Dexametasona-Ondansetron; DeDrO — Dexametasona-Droperidol-Ondansetron.

* Diferença estatística significativa, teste Exato de Fisher, $\mathrm{p}<0,05$.

+ Diferença estatística significativa, teste Exato de Fisher, $p<0,05$.

\section{DISCUSSÃO}

As náuseas e os vômitos pós-operatórios continuam sendo uma das causas mais comuns de complicação pós-anestésica.

Nesta pesquisa não foram utilizados o óxido nitroso e a neostigmina, assim como foi reduzido ao mínimo o uso de opióide no intra-operatório. O propofol não foi o hipnótico escolhido, em virtude de sua potencial atividade antiemética e interação com fármacos cronotrópicos negativos.

O tempo máximo de observação de náuseas e vômitos neste experimento foi de 36 horas, horário em que ocorreu a alta hospitalar. Estudo realizado por meio de entrevista, via telefone, após 24 a 48 horas e de questionários até o quinto dia da alta hospitalar, constatou que $35,7 \%$ dos pacientes apresentaram náuseas e vômitos ${ }^{10}$. Pesquisa empregando fármacos na profilaxia de náuseas e vômitos avaliou a presença desses efeitos adversos, as complicações e o grau de satisfação dos pacientes nas primeiras 48 horas ${ }^{11}$.

No presente estudo, a utilização de baixas doses de droperidol não acarretou nem disritmias cardíacas nem extensão do intervalo QT. Estudo eletrofisiológico em voluntários sadios sugeriu que seria improvável que baixas doses de droperidol, utilizadas como antiemético, acarretassem efeitos pró-disritmogênicos no intra-operatório ${ }^{12}$, o que é corroborado por trabalhos que avaliaram o efeito do droperidol no intervalo QT ${ }^{13-15}$

Estudos em seres humanos, utilizando dexametasona isolada ou associada ao ondansetron, em procedimento $\mathrm{ci}$ rúrgico por via laparoscópica, constataram a redução de náuseas e vômitos, recomendando seu uso de rotina ${ }^{16,17}$. Nesta pesquisa, a associação da dexametasona ao ondansetron não reduziu a incidência de náuseas e vômitos com relação ao seu uso isolado; entretanto, se associada também ao droperidol, ocorreu diminuição.

Pesquisas comparando a eficácia do droperidol, da metoclopramida e do ondansetron ${ }^{18,19}$ constataram que o ondansetron e o droperidol foram mais eficazes na profilaxia de náuseas e vômitos pós-operatórios. No presente estudo o ondansetron corroborou os estudos descritos, contudo o efeito do droperidol foi semelhante ao da metoclopramida. Nesta pesquisa, o ondansetron e as associações droperidol-ondansetron e dexametasona-droperidol-ondansetron foram mais eficazes na prevenção de náuseas, enquanto o 
ondansetron e a associação dexametasona-droperidolondansetron foram mais efetivos na prevenção de vômitos. A etiologia das náuseas e dos vômitos apresenta caráter multifatorial ${ }^{2,20}$, estando relacionada com quatro tipos de neurotransmissores que modulam a zona quimiorreceptora de gatilho, situada na área postrema: dopamina, serotonina, histamina e acetilcolina ${ }^{19}$. Os antieméticos são classificados conforme sua ação sobre os receptores farmacológicos e, normalmente, a administração isolada pode não ser adequada na profilaxia de náuseas e vômitos pós-operatórios. Alguns autores sugerem a associação de dois ou mais antieméticos para a obtenção de melhores resultados ${ }^{21-23}$, fato que foi observado neste estudo quando da utilização da associação dexametasona-droperidol-ondansetron.

Neste trabalho, o ondansetron isolado foi o agente mais eficaz na profilaxia de náuseas e vômitos pós-operatórios, sendo a associação dexametasona-droperidol-ondansetron uma boa opção em procedimentos cirúrgicos ginecológicos na pelve, pois apesar de ter o custo de três fármacos, tem também melhor eficácia na profilaxia de náuseas e vômitos, com maior grau de satisfação dos pacientes, não retardando as altas hospitalares, com conseqüente diminuição nos custos hospitalares.

Na profilaxia de náuseas e vômitos pós-operatórios, em procedimentos cirúrgicos ginecológicos, a associação dexametasona-droperidol-ondansetron e o ondansetron foram mais eficazes, não tendo sido observada qualquer reação adversa relacionada com os fármacos empregados.

\section{Comparative Study of Anti-Emetics and Their Association, in the Prevention of Postoperative Nausea and Vomiting in Patients Undergoing Gynecologic Surgeries}

Taylor Brandão Schnaider, M.D.; Antônio Mauro Vieira, TSA, M.D.; Antônio Carlos Aguiar Brandão, TSA, M.D.

\section{INTRODUCTION}

Specific odors, dehydration, pain, apprehension, and fear contribute for the development of postoperative nausea and vomiting ${ }^{1}$. Besides, nausea and vomiting do increase nosocomial costs by delaying hospital discharge, which demands unexpected hospitalizations, extended care by the medical team, and increases patient dissatisfaction ${ }^{2}$. Other complications include aspiration, aspiration pneumonia, water and electrolytes imbalance, esophageal rupture, dehiscence of sutures, and increase in intracranial pressure ${ }^{2}$.

The etiology of nausea and vomiting can be related to the patient (gender, age, weight, anxiety, history of postoperative nausea and vomiting, and smoking), anesthetic technique (nitrous oxide, halogenated, and opioids), and site and duration of the surgery ${ }^{3,4}$.

Female gender and gynecologic procedure are important risk factors, with an incidence around $70 \%$ of postoperative nausea and vomiting. For this reason, the search for the ideal anti-emetic for those patients continues ${ }^{2}$.

Metochlopramide and droperidol, antagonists of dopaminergic receptors, have been effective in the prevention and treatment of nausea and vomiting, but they can cause sedation, dysphoria, extrapyramidal syndrome, and spastic torticollis ${ }^{2,5}$.

Dexamethasone, a corticosteroid with anti-emetic action by an unknown mechanism, has also been effective in the prevention of postoperative nausea and vomiting, but it has higher efficiency in association with other anti-emetics ${ }^{2,5}$.

Ondansetron, a serotoninergic receptor antagonist (5-HT3 receptor), has had greater acceptation for the prevention of postoperative nausea and vomiting in patients with higher risk factors ${ }^{2,5}$.

Anti-emetic drugs with different mechanisms of action are synergistic when combined, resulting in greater efficacy in the prevention and treatment of postoperative nausea and vomiting ${ }^{2,5-9}$.

Due to the frequent episodes of postoperative nausea and vomiting and evolution of anesthetic and anti-emetic drugs, the objective of the present study was to compare several anti-emetics, administered as a single medication or in associations, in the prevention of those adverse effects in gynecologic surgeries.

\section{METHODS}

After approval by the Ethics Committee on Human Research of the Universidade do Vale do Sapucaí, patients were asked to sign an informed consent. Seventy female patients, ages 20 to 50 years, physical status ASA I and II, undergoing total abdominal hysterectomy with adnexectomy under general anesthesia associated with epidural block participated in this prospective an analytical clinic study.

Patients received oral diazepam $(10 \mathrm{mg}$ ) the day before surgery and oral midazolam (15 mg) 40 minutes before surgery. Patients were monitored with electrocardioscope, noninvasive blood pressure, pulse oximeter, and inspiratory and expiratory gas analyzer. After venipuncture with an $18 \mathrm{G}$ catheter, a solution containing midazolam (5 mg) and fentanyl $(50 \mu \mathrm{g})$ was administered.

Afterwards, the anti-emetic or its association, diluted in 500 $\mathrm{mL}$ of NS, was administered before the epidural puncture. The Metochlopramide Group (MG) received $20 \mathrm{mg}$ of metochlopramide; the Dexamethasone Group (DeG) received $8 \mathrm{mg}$; the Droperidol Group (DrG) received $1.25 \mathrm{mg}$; the Ondansetron Group (OG) received $8 \mathrm{mg}$; the Dexamethasone-Ondansetron Group (DeOG) received $8 \mathrm{mg}$ and $4 \mathrm{mg}$, respectively; the Droperidol-Ondansetron Group (DrOG) received $1.25 \mathrm{mg}$ and $4 \mathrm{mg}$, respectively; the Dexamethsone- 
Droperidol-Ondansetron Group (DeDrOG) received $8 \mathrm{mg}$, $0.625 \mathrm{mg}$, and $4 \mathrm{mg}$, respectively.

Epidural block was carried out with the patient in the sitting position, in the $L_{2}-L_{3}$ space, with a 15G Tuohy needle, and 20 $\mathrm{mL}$ of $0.75 \%$ ropivacaine were administered at $1 \mathrm{~mL} . \mathrm{s}^{-1}$.

Etomidate $\left(0.2 \mathrm{mg} \cdot \mathrm{kg}^{-1}\right)$, alfentanil $\left(30 \mu \mathrm{g} \cdot \mathrm{kg}^{-1}\right)$, and rocuronium $\left(0.6 \mathrm{mg}^{\mathrm{kg}}{ }^{-1}\right)$ were used for anesthetic induction, and isoflurane $(0.5 \%$ vol. to $3.0 \%$ vol. $)$ was used for maintenance. When clinical signs or hemodynamic responses suggesting inadequate levels of anesthesia (diaphoresis, tearing, hypertension, and tachycardia) were observed, intravenous alfentanil $(500 \mu \mathrm{g})$ was administered in intermittent doses.

Controlled ventilation was carried out with a low flow anesthesia system, allowing for the humidification and warming of inspired gases. Tidal volume varied from 8 to $10 \mathrm{~mL}^{\mathrm{kg}} \mathrm{kg}^{-1}$ and the respiratory rate was adjusted to maintain the expired pressure of carbon dioxide $\left(\mathrm{P}_{\mathrm{ET}} \mathrm{CO}_{2}\right)$ between 30 and $35 \mathrm{mmHg}$.

Blood pressure, heart rate, peripheral hemoglobin saturation $\left(\mathrm{SpO}_{2}\right)$, expired carbon dioxide $\left(\mathrm{P}_{\mathrm{ET}} \mathrm{CO}_{2}\right)$, and inspired concentration of isoflurane were recorded after installation of monitoring, epidural block, tracheal intubation, and every 15 minutes until the end of the surgery.
After the surgery, patients were transferred to the post-anesthetic care unit (PACU). Neuromuscular blockade antagonists were not used at the end of the procedure.

The incidence of nausea and vomiting was observed at $6 \mathrm{~h}$, $12 \mathrm{~h}, 24 \mathrm{~h}$, and $36 \mathrm{~h}$ after the surgery.

Analgesia consisted on the administration of dypirone, a pyrazolonic derivative, at $15 \mathrm{mg} \cdot \mathrm{kg}^{-1}$ every 4 hours after the surgery.

Analysis of Variance with Scheffé's test was used to analyze the anthropometric data; the Student $t$ test was used to analyze the length of the surgery; postoperative nausea and vomiting were analyzed by the Chi-square test and Fisher Exact test. $A \mathrm{p}<0.05$ was considered significant.

\section{RESULTS}

Analysis of Variance with Scheffé's test did not show statistically significant differences in the weight and age of the patients (Table I).

The Student $t$ test did not detect statistically significant differences in the length of surgery (Table II).

Patients did not require supplemental doses of opioids.

Table I - Anthropometric Data of Patients Included in the Study

\begin{tabular}{lcc}
\hline & Age (years) & Weight $(\mathrm{kg})$ \\
\hline Dexamethasone group $(\mathrm{n}=10)$ & $44.5 \pm 4.97$ & $68.7 \pm 8.68$ \\
Droperidol group $(\mathrm{n}=10)$ & $40.6 \pm 6.56$ & $68.4 \pm 7.86$ \\
Ondansetron group $(\mathrm{n}=10)$ & $41.9 \pm 6.36$ & $68.3 \pm 10.04$ \\
Metochlopramide group $(\mathrm{n}=10)$ & $41.8 \pm 6.35$ & $65.9 \pm 10.59$ \\
Droperidol-ondansetron group $(\mathrm{n}=10)$ & $43.7 \pm 5.33$ & $58.5 \pm 10.26$ \\
Dexametasona-ondansetron group $(\mathrm{n}=10)$ & $43.8 \pm 5.63$ & $63.8 \pm 12.04$ \\
Dexametasona-droperidol-ondansetron group $(\mathrm{n}=10)$ & $41.4 \pm 6.6$ & $60.7 \pm 5.22$ \\
\hline
\end{tabular}

Results expressed as Mean \pm SD.

Differences not statistically significant by Scheffé's test, $p>0.05$.

Table II - Duration of Anesthesia

\begin{tabular}{lc}
\hline & Duration $(\min )$ \\
\hline Dexamethasone group $(\mathrm{n}=10)$ & $100 \pm 28$ \\
Droperidol group $(\mathrm{n}=10)$ & $124 \pm 31$ \\
Ondansetron group $(\mathrm{n}=10)$ & $110 \pm 25$ \\
Metochlopramide group $(\mathrm{n}=10)$ & $108 \pm 26$ \\
Droperidol-ondansetron group $(\mathrm{n}=10)$ & $88 \pm 40$ \\
Dexametasona-ondansetron group $(\mathrm{n}=10)$ & $107 \pm 24$ \\
Dexametasona-droperidol-ondansetron group $(\mathrm{n}=10)$ & $96 \pm 26$ \\
\hline
\end{tabular}

Results expressed as Mean \pm SD.

Differences not statistically significant by the Student $t$ test, $\mathrm{p}>0.05$ 


\begin{tabular}{|c|c|c|c|c|c|c|c|}
\hline \multirow[b]{2}{*}{ Nausea } & \multicolumn{7}{|c|}{ Groups } \\
\hline & $\mathrm{Dr}$ & $\mathrm{De}$ & M & 0 & DrO & $\mathrm{DeO}$ & DeDrO \\
\hline Up to $6 \mathrm{~h}$ & 9 & 5 & 7 & 4 & 4 & 4 & 1 \\
\hline $12 \mathrm{~h}$ to $24 \mathrm{~h}$ & 3 & 3 & 5 & 1 & 1 & 2 & 1 \\
\hline $24 \mathrm{~h}$ to $36 \mathrm{~h}$ & 2 & 0 & 1 & 0 & 0 & 0 & 1 \\
\hline
\end{tabular}

Groups: Dr - Droperidol; De - Dexamethasone; M - Metochlopramide; O - Ondansetron; DrO - Droperidol-Ondansetron; DeO Dexamethasone-Ondansetron; DeDrO - Dexamethasone-Droperidol-Ondansetron.

* Statistically significant difference by the Chi-square test, $p<0.05$.

+ Statistically significant difference by the Chi-square test, $p<0.05$.

\# Statistically significant difference by the Chi-square test, $p<0.05$.

Table IV - Development of vomiting

\begin{tabular}{|c|c|c|c|c|c|c|c|}
\hline \multirow[b]{2}{*}{ Vomiting } & \multicolumn{7}{|c|}{ Groups } \\
\hline & $\mathrm{Dr}$ & $\mathrm{De}$ & $M$ & 0 & DrO & $\mathrm{DeO}$ & DeDrO \\
\hline Up to $6 \mathrm{~h}$ & 6 & 2 & 5 & 1 & 4 & 1 & 0 \\
\hline $6 h-12 h$ & 4 & 3 & 4 & 1 & 1 & 4 & 1 \\
\hline $12 h-24 h$ & 0 & 2 & 4 & 1 & 1 & 2 & 1 \\
\hline
\end{tabular}

Group: Dr - Droperidol; De - Dexamethasone; M - Metochlopramide; O - Ondansetron; DrO - Droperidol - Ondansetron; DeO -

Dexamethasone-Ondansetron; DeDrO - Dexamethasone-Droperidol-Ondansetron.

* Statistically significant difference by the Fisher Exact test, $p<0.05$.

+ Statistically significant difference by the Fisher Exact test, $p<0.05$.

The Chi-square test showed statistically significant differences in the incidence of nausea among the groups, as follows: between the Droperidol Group and the Dexamethasone, Ondansetron, Droperidol-Ondansetron, Dexamethasone-Ondansetron, and Dexamethasone-Droperidol-Ondansetron Groups; between the Metochlopramide and the Ondansentron, Droperidol-Ondansetron, and Dexamethasone-Droperidol-Ondansetron Groups; and between the Dexamethasone-Ondansetron and the DexamethasoneDroperidol-Ondansetron Groups (Table III).

The Fisher Exact test demonstrated significant differences between the following groups: Droperidol Group and Ondansetron and Dexamethasone-Droperidol-Ondansetron Groups; and between the Metochlopramide Group and the Ondansetron and Dexamethasone-Droperidol-Ondansetron Groups (Table IV).

\section{DISCUSSION}

Postoperative nausea and vomiting continue to be one of the most common post-anesthetic complications.

Neostigmine and nitrous oxide were not used in the present study, and the intraoperative use of opioids was reduced to a minimum. Propofol was not the hypnotic of choice due to its potential anti-emetic activity and interaction with negative chronotropic drugs.

In this study, 36 hours, the time of hospital discharge, was the maximal observation time for nausea and vomiting. A study conducted by telephone interviews from 24 to 48 after the discharge, and questionnaires up to the fifth day after hospital discharge demonstrated that $35.7 \%$ of patients developed nausea and vomiting ${ }^{10}$. A study with prophylactic drugs for nausea and vomiting evaluated the presence of those adverse effects, complications, and degree of patient satisfaction in the first 48 hours ${ }^{11}$.

In the present study, the use of low doses of droperidol did not cause cardiac arrhythmias or an increase in the QT interval. An electrophysiological study in healthy volunteers suggested that it would be unlikely that low doses of droperidol, used as an anti-emetic, had intraoperative proarrhythmogenic activity ${ }^{12}$, which was corroborated by studies that evaluated the effects of droperidol on the QT interval ${ }^{13-15}$.

Studies using dexamethasone alone or associated with ondansetron in laparoscopic surgeries in humans demonstrated a reduction in the incidence of nausea and vomiting, and recommended the routine use of those drugs ${ }^{16,17}$. In the 
present study, the association of dexamethasone and ondansetron did not reduce the incidence of nausea and vomiting when compared to dexamethasone alone; however, the addition of droperidol decreased their incidence.

A study comparing the efficacy of droperidol, metochlopramide, and ondansetron ${ }^{18,19}$ demonstrated that ondansetron and droperidol were more effective in the prophylaxis of postoperative nausea and vomiting. In the present study, the effects of ondansetron corroborated those studies; however, the effects of droperidol were similar to that of metochlopramide.

In the present study, Ondansetron and the association droperidol-ondansetron and dexamethasone-droperidol-ondansetron were more effective in the prophylaxis of nausea, while ondansetron and the association dexamethasonedroperidol-ondansetron were more effective in the prevention of vomiting. Nausea and vomiting have a multifactorial etiology ${ }^{2,20}$, being associated with four types of neurotransmitters that modulate the chemoreceptor trigger zone located in the area postrema: dopamine, serotonin, histamine, and acetylcholine ${ }^{19}$. Anti-emetics are classified according to their action on pharmacologic receptors and, usually, the administration of a single agent might not be adequate for prophylaxis of postoperative nausea and vomiting. Some authors suggest the association of two or more anti-emetics for better results 21-23, which was observed in the present study with the association dexamethasone-droperidol-ondansetron.

In the present study, ondansetron alone was the most effective agent in the prophylaxis of postoperative nausea and vomiting, and the association dexamethasone-droperidolondansetron is a good option for pelvic gynecological surgeries since, despite the cost of the three drugs, it is more effective in the prophylaxis of nausea and vomiting, has a greater degree of patient satisfaction, and does not delay discharge from the hospital, decreasing hospital costs.

The association dexamethasone-droperidol-ondansetron and ondansetron alone were more effective in the prevention of postoperative nausea and vomiting, and adverse reactions related to their use were not observed.

\section{REFERÊNCIAS - REFERENCES}

01. Watcha MF, White PF - Postoperative nausea and vomiting. Its etiology, treatment, and prevention. Anesthesiology, 1992;77: 164-184.

02. Lages N, Fonseca C, Neves A et al. - Náuseas e vômitos no pós-operatório: uma revisão do "pequeno-grande" problema. Rev Bras Anestesiol, 2005;55:575-585.

03 . Kenny GN - Risck factors for postoperative nausea and vomiting. Anaesthesia, 1994; 49:(Suppl)6-10.

04 . Lerman $\mathrm{J}-$ Surgical and patient factor envolved in postoperative nausea and vomiting. Br J Anaesth, 1992; 69:(Suppl)24S-32S.

05. Gan TJ - Postoperative nausea and vomiting - Can it be eliminated? JAMA, 2002; 287:1233-1236.

06. Yuen HK, Chiu JW - Multimodal antiemetic therapy and emetic risck profiling. Ann Acad Med Singapore, 2005; 34:196-205.
07. Abreu MP - Náuseas e vômitos - Antieméticos, em: Cangiani LM - Anestesia ambulatorial. São Paulo, Atheneu, 2001; 339357.

08. Ganem EM, Fabris P, Moro MZ et al. - Eficácia do ondansetron e da alizaprida na prevenção de náuseas e vômitos em laparoscopia ginecológica. Rev Bras Anestesiol, 2001;51:401-406.

09. Koivuranta M, Ala-Kokko TI, Jokela R et al. - Comparison of ondansetron and tropisetron combined with droperidol for the prevention of emesis in women with a history of postoperative nausea and vomiting. Eur J Anaesthesiol, 1999;16:390-395.

10. Carroll NV, Miederhoff P, Cox FM et al. - Postoperative nausea and vomiting after discharge from outpatient surgery centers. Anesth Analg, 1995;80:903-909.

11. Contreras-Domínguez V, Carbonell-Bellolio $P$ - Estudio clínico comparativo, randomizado y doble ciego entre droperidol, metoclopramida, tropisetrón, granisetrón e dexametasona para profilaxis antiemética en apendicectomia. Rev Argent Anestesiol, 2007;65:107-116.

12. Zhang $Y$, Luo Z, White PF - A model for evaluating droperidol's effect on the median QTc interval. Anesth Analg, 2004;98:13301335.

13. Nuttall GA, Eckerman KM, Jacob KA et al. — Does low-dose droperidol administration increase the risk of drug-induced QT prolongation and torsade de pointes in the general surgical population? Anesthesiology, 2007; 107:531-536.

14. Contreras-Domínguez V, Carbonell-Bellolio P - Profilaxia antiemética em cirurgia de abdome agudo. Estudo comparativo entre droperidol, metoclopramida, tropisetron, granisetron e dexametasona. Rev Bras Anestesiol, 2008;58:35-44.

15. White PF, Song D, Abrão J et al. - Effect of low-dose droperidol on the QT interval during and after general anesthesia: a placebo-controlled study. Anesthesiology, 2005;102:1101-1105.

16. Leksowski K, Peryga P, Szyca R - Ondansetron, metoclopramide, dexamethasone, and their combinations compared for the prevention of postoperative nausea and vomiting in patients undergoing laparoscopic cholecystectomy: a prospective randomized study. Surg Endosc, 2006; 20:878-882.

17. Elhakim M, Nafie M, Mahmoud $\mathrm{K}$ et al. - Dexamethasone $8 \mathrm{mg}$ in combination with ondansetron $4 \mathrm{mg}$ appears to be the optimaldose for the prevention of nausea and vomiting after laparoscopic cholecystectomy. Can J Anaesth, 2002;49: 922-926.

18. Abreu MP, Vieira JL, Silva IF et al. - Eficácia do ondansetron, metoclopramida, droperidol e dexametasona na prevenção de náuseas e vômitos após laparoscopia ginecológica em regime ambulatorial. Estudo comparativo. Rev Bras Anestesiol, 2006; 56:8-15.

19. Domino KB, Anderson EA, Polissar NL et al. - Comparative efficacy and safety of ondansetron, droperidol, and metoclopramide for preventing postoperative nausea and vomiting: a meta-analysis. Anesth Analg, 1999;88:1370-1379.

20. Schmidt A, Bagatini $A$ - Náuseas e vômitos pós-operatório: fisiopatologia, profilaxia e tratamento. Rev Bras Anestesiol, 1997; 47:326-334

21. Fugii $\mathrm{Y}$, Saitoh $\mathrm{Y}$, Tanaka $\mathrm{H}$ et al. - Granisetron/dexametasone combination for reducing nausea and vomiting during and after spinal anesthesia for cesarean section. Anesth Analg, 1999; 88:1346-1350.

22. Fugii $\mathrm{Y}$, Saitoh $\mathrm{Y}$, Tanaka $\mathrm{H}$ et al. - Prophylatic therapy with combined granisetron and dexametasone for the prevention of post-operative vomiting in children. Eur J Anaesthesiol, 1999; 16:376-379.

23. Mckenzie R, Uy NT, Riley TJ et al. - Droperidol/ondansetron combinations controls nausea and vomiting after tubal banding. Anesth Analg, 1996;83:1218-1222. 


\section{RESUMEN}

Schnaider TB, Vieira AM, Brandão ACA - Estudio Comparativo de Antieméticos y Sus Asociaciones, en la Prevención de Náusea y Vómito Postoperatorios, en Pacientes Sometidas a Procedimientos Quirúrgicos Ginecológicos.

JUSTIFICATIVA Y OBJETIVOS: La profilaxis de náusea y vómito postoperatorios fue objeto de muchos estudios. El objetivo de esta investigación fue comparar antieméticos y asociaciones en la prevención de náusea y vómito postoperatorios.

MÉTODO: Setenta pacientes, ASA I y II, fueron sometidas a procedimientos quirúrgicos ginecológicos, bajo bloqueo epidural asociado a la anestesia general. En el Grupo Metoclopramida (GM), se administró 20 mg; en el Grupo Dexametasona (GDe), se inyectó 8 mg; en el Grupo Droperidol (GDr) se administró 1,25 mg; en el Grupo Ondansetron (GO) se inyectó 8mg; en el Grupo Dexametasona-Ondansetron (GDeO) se administró respectivamente $8 \mathrm{mg}$ y 4mg; en el Grupo Droperidol-Ondansetron (GDrO) se inyectó 1,25 mg y 4 mg; en el Grupo Dexametasona-Droperidol-Ondansetron
(GDeDrO) se administró 8mg, 0,625 mg y 4mg. La presencia de náuseas y vómitos fue observada en los momentos de 6, 12, 24 y 36 horas después del término de la operación.

RESULTADOS: La incidencia total de episodios de náuseas fue de 4 en el GDeDrO, 6 en el GO, 6 en el GDrO, 11 en el GDe, 11 en el GDeO, 18 en el GM y 22 en el GDr. Al aplicar el test del Chi-cuadrado o el test de Fisher, se comprobó la diferencia estadística significativa entre el GDr y los grupos GDe, GDO, GDrO, GDeO, GDeDrO; entre el GM y los grupos GO, GDrO y GDeDrO; entre el GDeO y el grupo GDeDrO. La incidencia total de episodios de vómitos fue de 3 en el GO, 3 en el GDeDrO, 6 en el GDrO, 7 en el GDe, 7 en el GDeO, 10 en el GDr y 13 en el G Se comprobó así mismo, la diferencia estadística significativa entre el GDr y los grupos GO y GDeDrO; entre el GM y los grupos GO y GDeDrO.

CONCLUSIONES: La asociación dexametasona-droperidol-ondansetron y el ondansetron fueron más eficaces en la profilaxis de náuseas y vómitos. 